\title{
Re: The Relationship Between Irritable Bowel Syndrome, Functional Dyspepsia, Chronic Fatigue and Overactive Bladder Syndrome: A Controlled Study 6 Years After Acute Gastrointestinal Infection
}

\author{
Heidi Brown ${ }^{\mathrm{a}}$ and Rufus Cartwright ${ }^{\mathrm{b},{ }^{*}}$ \\ aSchool of Medicine and Public Health, University of Wisconsin, Madison, WI, USA \\ ${ }^{b}$ Department of Epidemiology and Biostatistics, Imperial College London, London, UK
}

\section{Experts' summary}

Following up patients affected by an outbreak of giardiasis in Bergen, Norway, in 2004, this elegant study identified a higher prevalence of overactive bladder $(\mathrm{OAB})$ after previous confirmed giardiasis compared with matched controls (18.7\% [ $n=716]$ vs $13.6 \%$ [ $n=833]$; $p=0.007)$. The authors also explored interactions between OAB and irritable bowel syndrome, functional dyspepsia, and chronic fatigue. They postulated that the apparent association between $\mathrm{OAB}$ and giardiasis can be ascribed to comorbid functional disorders because the prevalence of $\mathrm{OAB}$ among exposed versus control participants without comorbid disorders was similar (12.4\% vs $10.9 \%, p=0.51)$, and the risk of OAB was more than two-fold increased with any functional comorbid disorder.

\section{Experts' comments}

Although most management strategies for OAB target the bladder, these authors suggested instead that the observed overlap between $\mathrm{OAB}$ and other functional disorders might arise either because of cross-sensitization of common neural pathways between gut and bladder or because of a tendency to overinterpret somatic sensations. We have compelling evidence of an association between affective disorders and lower urinary tract symptoms [1], and this study provides a further reminder of the importance of a holistic approach to OAB.

A second line for future investigation may explore the relationship between Giardia infection and the urinary microbiome. Giardia infection causes a profound transient shift in the fecal flora, and some changes may persist beyond resolution of clinical infection [2]. The fecal and urinary microbiota are intimately linked [3], and recent research has demonstrated differences in the urinary microbiota of women with and without $\mathrm{OAB}$, using both $16 \mathrm{~S}$ ribosomal RNA sequencing and expanded quantitative urine culture techniques [4]. In a murine model of Giardia, the bacterial strains that penetrated the gut mucosa in the

*Corresponding author. Department of Epidemiology and Biostatistics, Imperial College London, Norfolk Place, London W2 1PG, UK. rufus.cartwright@gmail.com (R. Cartwright).

Conflicts of interest: The authors have nothing to disclose. 
postinfective phase included Bacillus, Lactobacillus, Staphylococcus, and Phenylobacterium [2]. Two of these four bacteria, Staphylococcus and Lactobacillus gasseri, were identified more frequently in a US study of the urinary microbiome of women with OAB [4]. It is certainly possible that the apparent epidemiologic association between Giardia exposure and $\mathrm{OAB}$ is mediated only by relationships with other functional disorders, but it is also possible that Giardia exposure has a persistent impact on the fecal and urinary microbiota, even $6 \mathrm{yr}$ following exposure. This study provides further support for chronic bacterial infection as one precipitant of $\mathrm{OAB}$, and further research should explore this possibility and its implications for treatment.

\section{References}

1. Vrijens D, Drossaerts J, van Koeveringe G, Van Kerrebroeck P, van Os J, Leue C. Affective symptoms and the overactive bladder - a systematic review. J Psychosom Res. 2015; 78:95-108. [PubMed: 25499886]

2. Chen TL, Chen S, Wu HW, et al. Persistent gut barrier damage and commensal bacterial influx following eradication of Giardia infection in mice. Gut Pathog. 2013; 5:26. [PubMed: 23991642]

3. Jakobsen L, Garneau P, Bruant G, et al. Escherichia coli urinary tract infection a zoonosis? Proof of direct link with production animals and meat. Eur J Clin Microbiol Infect Dis. 2012; 31:1121-9. [PubMed: 22033854]

4. Pearce MM, Hilt EE, Rosenfeld AB, et al. The female urinary microbiome: a comparison of women with and without urgency urinary incontinence. mBio. 2014; 5:e01283-1314. [PubMed: 25006228] 